\title{
Ambulatory abdominal inductance plethysmography: towards objective assessment of abdominal distension in irritable bowel syndrome
}

\author{
M J V Lewis, B Reilly, L A Houghton, P J Whorwell
}

\begin{abstract}
Background-Abdominal distension is one of the cardinal features of irritable bowel syndrome (IBS) with patients often ranking it as more intrusive than their pain or bowel dysfunction. If this symptom could be quantified accurately and reliably it would provide, for the first time, an objective way of assessing this disorder. Using the principle of inductance plethysmography we have developed a microprocessor based system capable of recording even minimal changes in girth over 24 hours and we describe its validation and establish normal ranges.

Methods-Twenty four hour recordings were made in $\mathbf{2 0}$ healthy female volunteers (aged 23-58 years) to assess changes in abdominal girth with respect to time, posture, meals, and sleep. Normal daily activity was encouraged and meals were standardised. The feeling of abdominal distension was also assessed using a visual analogue scale.
\end{abstract}

Findings-Measurement of abdominal girth using abdominal inductance plethysmography (AIP) at a static point in time showed a close relationship with that measured using a tape measure (rho=0.8910, $\quad \mathbf{p}<0.001)$. Furthermore, girth was significantly greater both in the sitting and standing postures compared with lying $(p<0.01)$. Measurement over a 24 hour period showed that girth was significantly greater at the end compared with the beginning of the day, and ingestion of a standard meal also increased its value $(p<0.05)$. Sleep was associated with a reduction in girth $(\mathrm{p}<0.05)$.

Conclusions-AIP promises to add an objective dimension to the assessment of IBS which could prove invaluable in clinical trials. Furthermore, it should aid research into the pathogenesis of this symptom for which no satisfactory explanation has yet emerged.

(Gut 2001;48:216-220)

Keywords: abdominal distension; irritable bowel syndrome

Correspondence to: Dr L A Houghton, Department of Medicine, University Hospital of South Manchester, Nell Lane, West Didsbury, Manchester M20 2LR, UK.

lahoughton@man.ac.uk

Accepted for publication 8 August 2000

Therapeutic trials into new pharmacological have always been severely hampered by the lack of any objective measure of symptoms. This situation is further compounded by the fact that none of the symptoms of the disorder can be explained by any identifiable structural, physiological, or laboratory abnormality.

One of the most common and intrusive symptoms of IBS is abdominal distension ${ }^{1}$ (often referred to as bloating ${ }^{2}$ ), which has been reported to occur more than once a week in $86 \%$ of patients. ${ }^{3}$ It appears to affect both patients with diarrhoea and constipation predominant bowel habits ${ }^{4}$ and is more common in females than males. ${ }^{5}$ It is noteworthy that $30 \%$ of apparently healthy individuals also describe episodes of distension on a regular basis, ${ }^{6}$ raising the possibility that it may be a normal phenomenon that is either more exaggerated or differently perceived in patients with IBS. Sufferers typically report a sense of progressive abdominal distension throughout the day which is often worse after meals and requires loosening of clothes by the evening. ${ }^{3}$ It generally improves overnight. ${ }^{3}$ The cause of abdominal distension is incompletely understood, although the obvious explanation of accumulation of gas is unlikely. ${ }^{7}$

Some studies have attempted to quantify abdominal distension using a tape measure ${ }^{78}$ but this technique is bound to be influenced by either patient or observer, or both. In addition, this method only allows static measurements of abdominal girth at intermittent points in time. If distension could be accurately recorded over prolonged periods of time, this would be a major advance allowing, for the first time, the objective measurement of a feature of IBS. This might not only enhance therapeutic research but also lead to a better understanding of this symptom.

The aim of this study was therefore to create an unobtrusive, automated, ambulatory device to allow fully objective monitoring of abdominal girth in the subject's usual posture and environment, and to establish, using this technique, normal variations in abdominal girth.

\section{Materials and methods}

ABDOMINAL INDUCTIVE PLETHYSMOGRAPHY (AIP) The cross sectional area of the thorax can be measured using a technique called inductance plethysmography. ${ }^{9}$ This is based on the principle that a loop of wire forms an inductor, the inductance of which is dependent on the area enclosed by the loop. The equipment consists of a band of elasticated fabric (approximately $8.5 \mathrm{~cm}$ wide) into which is sewn a wire in zig zag fashion to allow for expansion (Respitrace
Abbreviations used in this paper: IBS, irritable bowel syndrome; AIP, abdominal inductance plethysmography; AP, anteroposterior; L, lateral. 


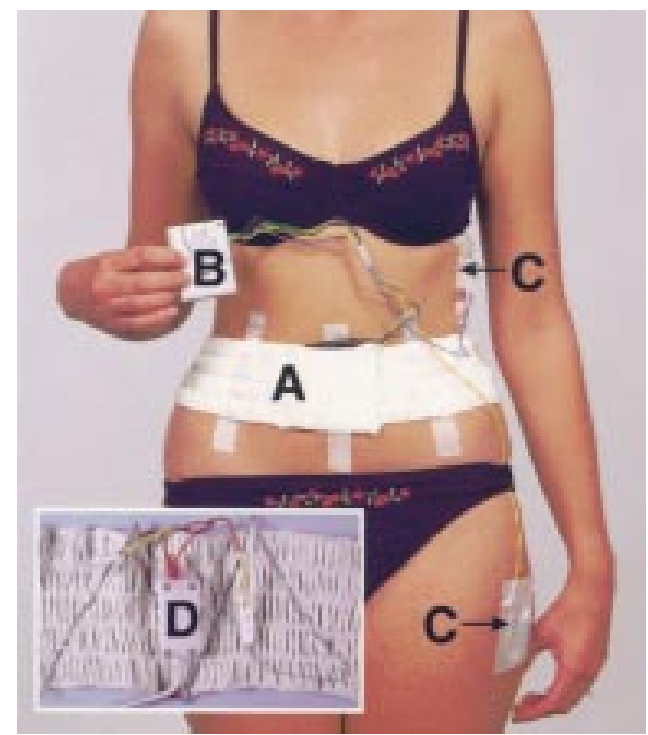

Figure 1 Photograph of a subject wearing the equipment. $A$, the belt; $B$, data logger; and $C$, mercury tilt switches. Insert shows a close up of the belt revealing the wire sewn in a zig zag fashion and connected to an oscillator (D) which is secured under the belt.

inductive sensor; Studley Data Systems, Oxford, UK). Attached to the wire is a small electronic circuit unit taped next to or under the band. This incorporates an inductor in a resonant circuit whose output frequency varies with the area enclosed by the band. This technology has obvious potential for measuring abdominal girth but current systems use bulky mains operated recording equipment linked to a computer which is therefore unsuitable for ambulatory situations. We have designed an ambulatory system consisting of a small battery operated microprocessor "data logger" (weight $90 \mathrm{~g}$, dimensions $72 \mathrm{~mm} \times 55 \mathrm{~mm} \times 18 \mathrm{~mm}$ ) which records and stores the average frequency of the oscillator circuit for 30 seconds every minute. The same unit simultaneously records posture (standing, sitting, lying) via sealed mercury tilt switches (ASSEMtech Europe Ltd, Essex, UK) taped to the subject's chest and thigh (fig 1).

CALIBRATION

Four belts were used in this study $(70,80,90$, and $100 \mathrm{~cm}$ ) and each was calibrated by the use of a variable frame the size of which can be precisely adjusted using a computer.

SUBJECTS

Twenty healthy female volunteers (aged 23-58 years, mean 39.4 years) participated in the study. No subject had experienced any chronic gastrointestinal or gynaecological disorder and none had taken medication liable to alter gastrointestinal function. Smoking, alcohol consumption, and ingestion of all food and drinks containing caffeine were prohibited during the study and for the preceding 24 hours. Approval was obtained from the South Manchester ethics committee and all subjects gave fully informed consent.

Based on pilot data, this study on 20 healthy volunteers had $80 \%$ power of detecting a change in girth of $1.2 \mathrm{~cm}$.

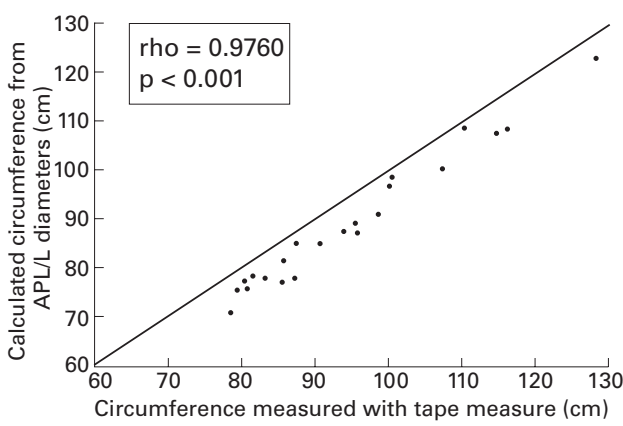

Figure 2 Relationship between abdominal circumference calculated from anteroposterior $(A P)$ and lateral $(L)$ diameters and that obtained from the tape measure (line represents line of equality).

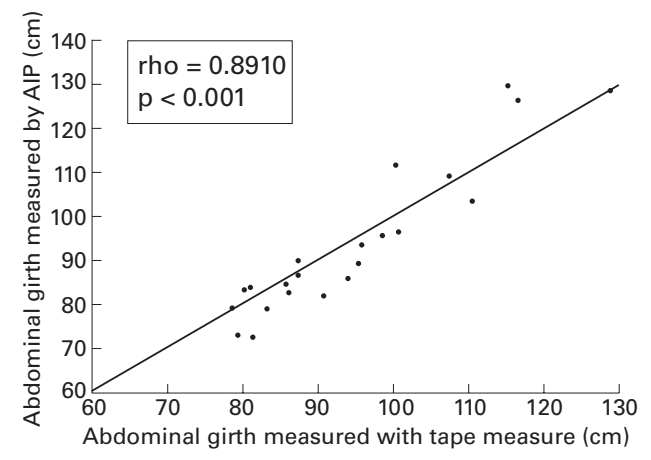

Figure 3 Relationship between abdominal girth measured by abdominal inductance plethysmography (AIP) and that obtained with the tape measure (line represents line of equality).

PROTOCOL

After an overnight fast, volunteers attended the laboratory no later than 1100 hours (day 1). Abdominal circumference (tape measure) and the anteroposterior (AP) and lateral (L) diameters (callipers) were measured in the standing position at the level of the umbilicus. The belt was then positioned at the same level and securely taped to the back to prevent slippage. Tilt switches were secured to the chest and thigh. Each volunteer was given a light packed lunch comprising a cheese sandwich (Deep Fill Ploughman's sandwich; Ginster's, Callington, UK), a medium apple, and a $288 \mathrm{ml}$ carton of low sugar blackcurrant cordial (Ribena; SmithKline Beecham, Brentford, UK) which was to be eaten at 1230 hours (providing in total 647 kcal energy, $20.6 \mathrm{~g}$ protein, $71.8 \mathrm{~g}$ carbohydrate, $28.2 \mathrm{~g}$ fat, and $11.9 \mathrm{~g}$ fibre). They returned to the unit between 1620 and 1820 hours and were given a standard evening meal of vegetarian lasagne (300 g, Tesco, Cheshunt, UK), sweetcorn (100 g, Pillsbury, Uxbridge, UK), bread roll (67 g, Tesco), low fat strawberry yoghurt (150 g, Tesco), and $400 \mathrm{ml}$ water, all of which contained $719 \mathrm{kcal}$ energy, $27.9 \mathrm{~g}$ protein, $107.1 \mathrm{~g}$ carbohydrate, $19.4 \mathrm{~g}$ fat, and $6.9 \mathrm{~g}$ fibre. These meals were designed to provide the average daily nutrient intake for females in the UK. Only sips of water were allowed between the standard meals and volunteers were encouraged to pursue as much as possible their normal daily activities. The volunteers reattended the lab the following morning (day 2) to have the belt removed. 
Table 1 Abdominal girth measured using abdominal inductance plethysmography in various standardised postures (cm)

\begin{tabular}{llllll}
\hline & Beginning of day 1 & Immediately pre-meal & Immediately post-meal & 1 hour post-meal & Beginning of day 2 \\
\hline Lying & 81.23 & 81.93 & 82.80 & 83.11 & 82.12 \\
\multirow{2}{*}{ Sitting } & $(79.46,88.78)$ & $(75.51,89.65)$ & $(76.72,91.74)$ & $(76.33,92.83)$ & $(73.19,92.54)$ \\
& $85.80^{\mathrm{a}}$ & $86.65^{\mathrm{a}}$ & $85.82^{\mathrm{ac}}$ & $87.09^{\mathrm{ac}}$ & $83.80^{\mathrm{a}}$ \\
Standing & $(78.15,94.83)$ & $(78.06,93.84)$ & $(79.75,95.59)$ & $(79.80,96.46)$ & $(75.34,94.44)$ \\
& $85.65^{\mathrm{a}}$ & $88.10^{\mathrm{ab}}$ & $89.46^{\mathrm{ac}}$ & $89.08^{\mathrm{ac}}$ & $88.92^{\mathrm{a}}$ \\
& $(78.54,98.78)$ & $(78.03,97.56)$ & $(79.06,98.97)$ & $(79.06,99.62)$ & $(76.18,104.61)$
\end{tabular}

Results are median (interquartile range).

${ }^{a}$ Compared with lying, $\mathrm{p}<0.01$; ${ }^{\mathrm{b}}$ compared with sitting, $\mathrm{p}<0.01$; ${ }^{\mathrm{c}}$ compared with immediately pre-meal, $\mathrm{p}<0.05$.

To investigate the effects of different postures on abdominal girth, volunteers were asked to lie, sit, and stand (three minutes each, consecutively) at the start of the study, immediately before and after the standard evening meal, one hour after the standard evening meal, and the following morning. These are referred to in the results section as static measurements. In addition, at each of these time points, they were asked to indicate on a visual analogue scale $(0-100 \mathrm{~mm}$, were $100 \mathrm{~mm}$ is maximum) their perception of their degree of abdominal distension.

DATA ANALYSIS

To convert the cross sectional area recorded by the equipment into a more conventional circumferential measurement, we assumed the abdomen to be an ellipse in cross section. This was confirmed by calculating the abdominal circumference from the AP and L diameters and comparing this value with that obtained from the tape measure. Although the calculated circumference was slightly less than that obtained using the tape measure (median difference (interquartile range, IQR) $-5.2 \mathrm{~cm}$ $(-6.4,-3.0))$, the ranking of subjects with respect to abdominal circumference (measured using $\mathrm{AP} / \mathrm{L}$ diameters and tape measure) showed a close relationship between the two parameters (rho $=0.9760, \mathrm{p}<0.001$ ) (fig 2). Furthermore, any effect that different sizes and shapes of abdomens might have on our elliptical assumption was assessed by calculating the eccentricity of the ellipse (based on AP to L

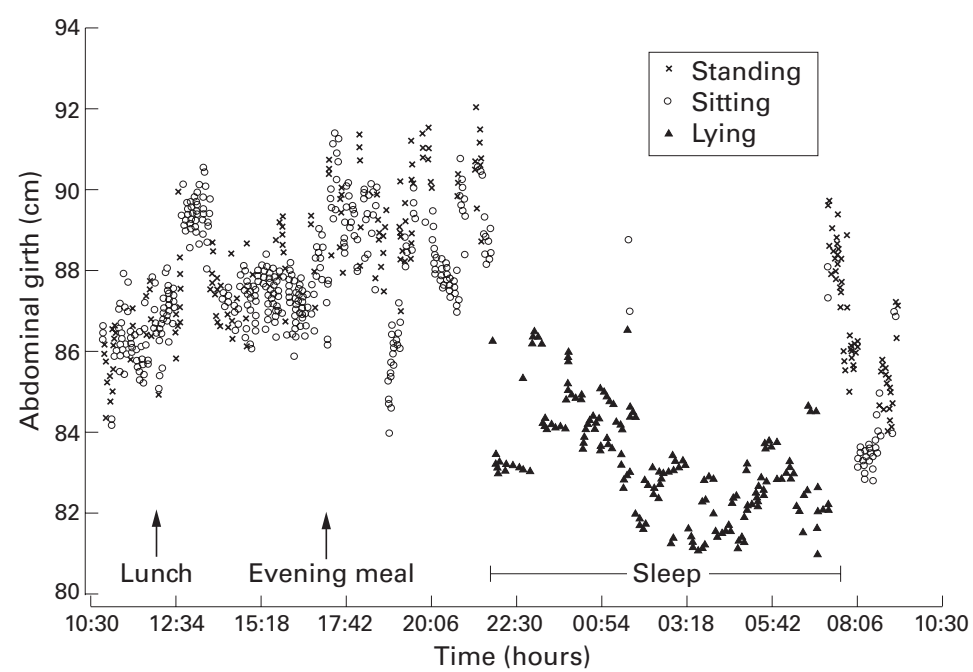

Figure 4 Typical recording of abdominal girth over 24 hours in a normal healthy volunteer. Note the gradual increase in girth during the day, increase with meal ingestion, and slow reduction in girth during sleep. diameter). This showed that even with a wide range of sizes and shapes of abdomens (circumference, median (IQR); $88.90 \mathrm{~cm}$ $(81.85,100.18))$ there was little variation in the eccentricity (mean 0.70 (SD 0.06)).

Using the derived circumferential data, the 24 hour recordings were then divided into daytime standing and sitting data, and night time lying data. Recordings in the standing and sitting postures were then averaged over one hour epochs (i) at the start of recording, (ii) immediately before the standard meal, (iii) immediately after the standard meal, (iv) immediately before going to bed, and (v) at the end of the recording on the morning of day 2 . Data obtained in the lying posture were averaged for the 30 minutes immediately after going to bed and again before rising.

STATISTICAL ANALYSIS

Changes in abdominal girth were assessed with Wilcoxon's signed rank test and correlations between changes in abdominal girth and subjective bloating scores were performed using Spearman's test.

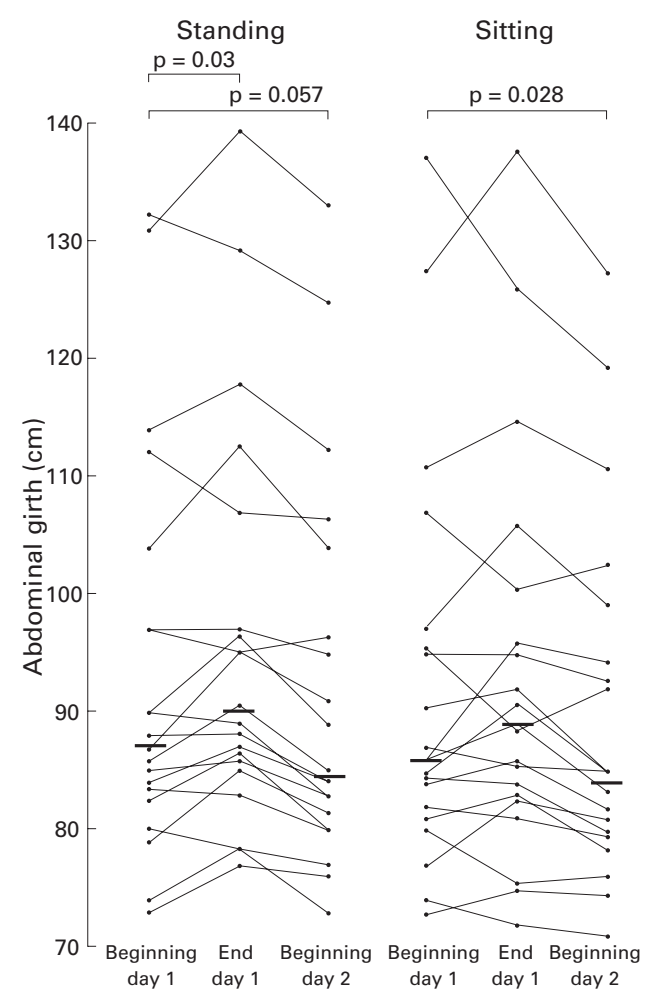

Figure 5 Abdominal girth measured by abdominal inductance plethysmography at the beginning of day 1, at the end of day 1, and at the beginning of day 2 for standing and sitting postures. Bars represent median values. 


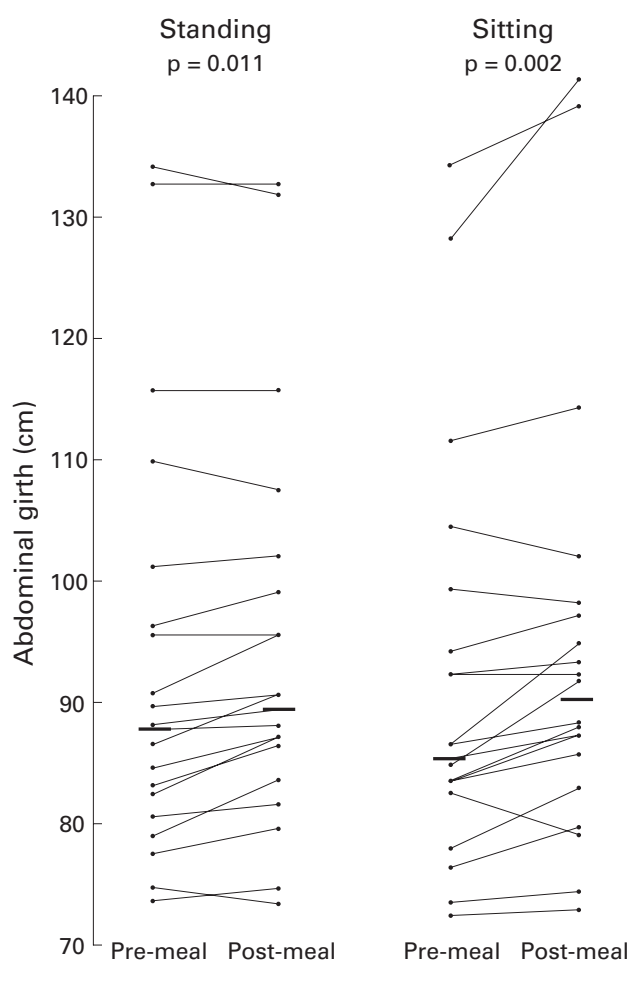

Figure 6 Abdominal girth measured by abdominal inductance plethysmography before and after the standard evening meal for standing and sitting postures. Bars represent median values.

\section{Results}

VALIDATION

Abdominal girth values measured by AIP in the standing posture during the first 10 minutes of the study (median (IQR), $87.11 \mathrm{~cm}$ $(83.16,103.27))$ were similar to those obtained with the tape measure $(88.90 \mathrm{~cm}(81.85$, $100.18)$, median difference $-0.9 \mathrm{~cm}(-4.8$, 3.4)) and the ranking of subjects with respect to girth using both of these measures showed a close relationship between the two (rho $=0.8910, \mathrm{p}<0.001$ ) (fig 3).

\section{EFFECT OF POSTURE}

It can be seen from table 1 that at each of the various static time points throughout the study, abdominal girth was significantly greater in the sitting and standing postures compared with the lying postures $(\mathrm{p}<0.01)$. There were no significant differences in girth between the sitting and standing postures, with the exception of minor differences pre-meal (table 1).

\section{TWENTY FOUR HOUR RECORDING}

Figure 4 shows a typical recording of abdominal girth over 24 hours in a normal healthy volunteer. Abdominal girth was significantly greater at the end compared with at the beginning of the day (day 1), for the standing (median increase (IQR), $3.2 \mathrm{~cm}(-1.0,5.3)$; $\mathrm{p}=0.03)$ but not sitting $(1.4 \mathrm{~cm}(-2.0,5.2)$; $\mathrm{p}=0.411$ ) postures (figs 4,5$)$. There was no significant difference in girth immediately before the standard evening meal compared with the beginning of the day for both the standing $(88.1 \mathrm{~cm}(81.7,100.3) v 87.3 \mathrm{~cm}$ $(82.8,102.1))$ and sitting $(86.3 \mathrm{~cm}(83.0,98.8)$

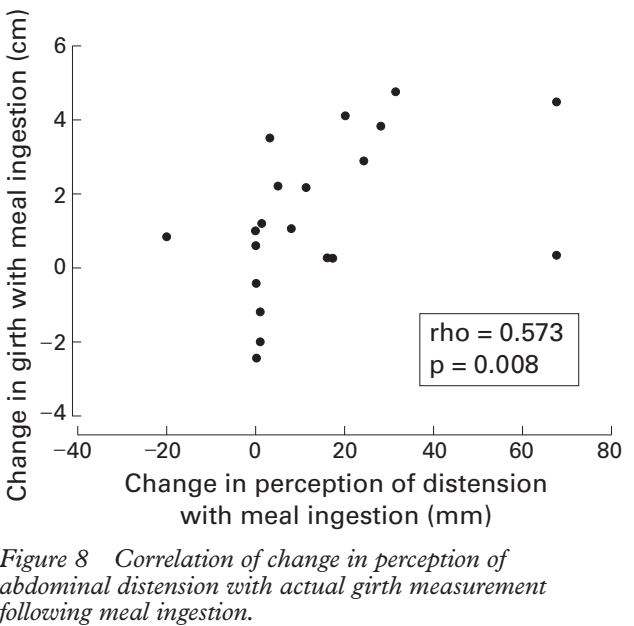

$v 86.3 \mathrm{~cm}(81.0,97.1))$ postures. However, ingestion of the standard meal increased girth in both the standing $(1.1 \mathrm{~cm}(0.4,3.4)$; $\mathrm{p}=0.011)$ and sitting $(2.7 \mathrm{~cm}(0.8,4.9)$; $\mathrm{p}=0.002$ ) postures (figs 4,6 ). Similar increases were seen when comparing static time points (table 1). Interestingly, girth was lower the following morning (day 2) compared with the previous morning (day 1) (standing: $-1.3 \mathrm{~cm}$ $(-3.4,0.2), \mathrm{p}=0.057$; sitting: $-2.5 \mathrm{~cm}(-4.3$, 0.2 ), $\mathrm{p}=0.028$ ) (fig 5).

The effect of a night's sleep was to reduce abdominal girth by $1.2 \mathrm{~cm}(-0.3,2.9)$ $(\mathrm{p}=0.028)$, as reflected by the measurements 30 minutes after retiring to bed and 30 minutes before rising (fig 7). Furthermore, this change occurred slowly over the course of the night, as can be seen from fig 4 .

PERCEPTION OF ABDOMINAL DISTENSION

Compared with the beginning of day 1 , the volunteers' perceptions of their distension had significantly increased by the time they arrived back at the laboratory for their standard evening meal (median (IQR), $4.0 \mathrm{~mm}$ (1.0, 8.8) $v 18.5 \mathrm{~mm}(3.8,40.0) ; \mathrm{p}=0.001)$. This increased further with consumption of the standard meal $(27.0 \mathrm{~mm}(11.0,59.0) ; \mathrm{p}=0.003$ compared with pre-meal) and was still significantly elevated one hour after ingestion (21.5 $\mathrm{mm}(8.8,51.3) ; \mathrm{p}=0.014$ compared with pre-meal). There was no difference in the subjective measure of distension between the beginning of study days 1 and 2 (beginning of day $2,5.0 \mathrm{~mm}(0,8.8))$.

PERCEPTION AND ITS RELATIONSHIP TO GIRTH There was a direct correlation between the volunteer's change in perception of their distension with meal ingestion and the actual change in girth (standing data, rho $=0.573 ; \mathrm{p}=0.008$ ) (fig 8). There were no other correlations between change in perception and abdominal girth.

\section{Discussion}

This study has clearly demonstrated that AIP can measure even small changes in girth accurately and reliably over 24 hours in healthy individuals. It has also shown that girth changes during the day in normal healthy indi- 
viduals and seems to follow a particular pattern-namely, that it increases as the day progresses, is more pronounced after meals, and subsides at night. In addition, the normal individual's perception of their girth after meals shows a good correlation with actual measurements.

Our results using static AIP measurements show that although girth in the standing and sitting positions is similar, there was a significant reduction when a subject adopted the lying posture. However, on continuous monitoring the changes in standing data were not always reflected in the sitting data and this is probably an indication of the variability of the sitting position (for example, upright $v$ slouched). These observations indicate that in future studies the belt will not be able to be worn alone and that some form of monitoring of posture will still need to be incorporated.

It is of interest that girth on the following morning (day 2) was less than that recorded at the start of the study. This might be explained by the fact that as a result of being standardised, the study day may have differed from the pre-study day in terms of a number of possible distension provoking factors. These could have included physical activity, different types of food and drink, snacks, or even subtle changes in stress levels.

Thus AIP should allow the further assessment of factors which might affect girth in normal subjects (dietary constituents, stress, gender, etc) and the investigation of the relationship between this objective measurement and the subjective symptom of abdominal distension in patients with functional bowel disorders. For instance, is distension in IBS a purely mechanical phenomenon or is it a disor- der of perception or a combination of the two. It is possible that distension may not be entirely symmetrical and occur at slightly different sites in different individuals. However, the belt should be sufficiently wide to capture most, if not all, of this change in the majority of situations. If, in the future, it was felt necessary to study the distribution of bloating (upper $v$ lower abdomen) in different functional syndromes then there is no reason why this could not be assessed by wearing two belts simultaneously.

In conclusion, this technology should help elucidate the symptom of distension in irritable bowel syndrome and also be invaluable as an objective measure in the field of clinical trials.

Dr MJV Lewis was supported by an educational grant from SmithKline Beecham Pharmaceuticals. We would also like to thank Mrs J Morris, Head of Medical Statistics, for her advice.

1 Maxton DG, Morris JA, Whorwell PJ. Ranking of symptoms by patients with irritable bowel syndrome. $B M \mathcal{F}$ 1989;299:1138.

2 Thompson WG, Creed F, Drossman DA, et al. Functional bowel disorders and functional abdominal pain. Gastroenbowel disorders and fun

3 Maxton DG, Whorwell PJ. Abdominal distension in irritable bowel syndrome: the patient's perspective. Eur $\mathcal{F}$ irritable bowel syndrome: the patient

4 Talley NJ, Zinsmeister AR, Van Dyke C, et al. Epidemiology of colonic symptoms and irritable bowel syndrome. Gastroenterology 1991;101:927-34.

5 Heaton KW, O'Donnell LJD, Braddon FEM, et al. Symptoms of irritable bowel syndrome in a British urban community: consulters and non-consulters. Gastroenterology 1992;102:1962-7.

6 Thompson WG, Heaton KW. Functional bowel disorders in apparently healthy people. Gastroenterology 1980;79:283-8.

7 Maxton DG, Martin DF, Whorwell PJ, et al. Abdominal distension in female patients with irritable bowel into 1991;332:662-4.

8 Sullivan SN. A prospective study of unexplained visible abdominal bloating. NZ Med f 1994;107:428-30.

9 Watson HL, Poole DA, Sackner MA. Accuracy of respiratory inductive plethysmographic cross-sectional areas. $\mathcal{F}$ Appl Physiol 1988;65:306-8. 